7th International Workshop on Astronomy and

Relativistic Astrophysics (IWARA 2016)

International Journal of Modern Physics: Conference Series

Vol. 45 (2017) 1760035 (8 pages)

(C) The Author(s)

DOI: $10.1142 / \mathrm{S} 2010194517600357$

\title{
Rotation-Driven Phase Transitions in the Cores of Pulsars
}

\author{
Richard D. Mellinger Jr, William Spinella \\ Department of Physics \\ San Diego State University \\ 5500 Campanile Drive, 92182 San Diego, CA, USA \\ imasillypirate@gmail.com,spinella@rohan.sdsu.edu \\ Fridolin Weber \\ Department of Physics \\ San Diego State University \\ 5500 Campanile Drive, 92182 San Diego, CA, USA \\ Center for Astrophysics and Space Sciences \\ University of California \\ San Diego, 92093 La Jolla, CA, USA \\ fweber@mail.sdsu.edu \\ Gustavo A. Contrera \\ Consejo Nacional de Investigaciones Científicas y Técnicas (CONICET) \\ Godoy Cruz 2290, 1425 Buenos Aires, Argentina \\ Universidad Nacional de La Plata (UNLP) \\ Calle 51 E 124-125, Ensenada, Argentina La Plata, Argentina \\ contrera@fisica.unlp.edu.ar \\ Milva Orsaria \\ Department of Physics \\ San Diego State University \\ 5500 Campanile Drive, 92182 San Diego, CA, USA \\ Consejo Nacional de Investigaciones Científicas y Técnicas (CONICET) \\ Godoy Cruz 2290, 1425 Buenos Aires, Argentina \\ Universidad Nacional de La Plata (UNLP) \\ Calle 51 E 124-125, Ensenada, Argentina La Plata, Argentina \\ morsaria@fcaglp.unlp.edu.ar
}

Published 15 August 2017

In this paper, we discuss the impact of rotation on the particle composition of rotating neutron stars (pulsars). Particular emphasis is put on the formation of quark matter during stellar spin-down, driven by continuous gravitational compression. Our study is

This is an Open Access article published by World Scientific Publishing Company. It is distributed under the terms of the Creative Commons Attribution 4.0 (CC-BY) License. Further distribution of this work is permitted, provided the original work is properly cited. 


\title{
R. D. Mellinger Jr et al.
}

\begin{abstract}
based on modern models for the nuclear equation of state whose parameters are tightly constrained by nuclear data, neutron star masses, and the latest estimates of neutron star radii.
\end{abstract}

Keywords: Dense Matter, Equation of State, Neutron Stars, Pulsars.

PACS numbers: 25.75.Nq, 26.60.-c, 26.60.Kp, 97.60.Gb

\section{Introduction}

Most of the neutron star calculations reported in the literature have been performed for non-rotating, spherically symmetric stellar configurations, whose properties are uniquely determined by the Oppenheimer-Volkoff equation ${ }^{1,2}$. However a steady increase in the number of observed rotating neutron stars with rotational periods in the millisecond range has renewed considerable interest in the influence and implications of rapid rotation on the properties of neutron stars. Details about these equations of state (EoS) can be found in the recent paper by Mellinger et al. ${ }^{3}$ and in the contribution of Contrera et al. ${ }^{4}$ contained elsewhere in this volume.

\section{Rotating Neutron Stars in the Framework of General Relativity}

The fact that rotation deforms neutron stars, stabilizes them against collapse, and drags along the local inertial frames inside and outside of them so that they corotate with the stars, renders the construction of models of rotating neutron stars rather complicated. A suitable ansatz for the line element (that is, the components of the metric tensor) has the form ${ }^{2}$

$$
d s^{2}=-e^{2 \nu} d t^{2}+e^{2 \psi}(d \phi-\omega d t)^{2}+e^{2 \mu} d \theta^{2}+e^{2 \lambda} d r^{2}
$$

where $\nu, \psi, \mu$ and $\lambda$ denote metric functions and $\omega$ is the angular velocity of the local inertial frames. All these quantities depend on the radial coordinate $r$, the polar angle $\theta$, and implicitly on the star's angular velocity $\Omega$. The metric functions and the frame dragging frequencies are to be computed from Einstein's field equation,

$$
R^{\kappa \sigma}-\frac{1}{2} R g^{\kappa \sigma}=8 \pi T^{\kappa \sigma}
$$

where $T^{\kappa \sigma}=T^{\kappa \sigma}(\epsilon, P(\epsilon))$ denotes the energy momentum tensor of the stellar matter, whose equation of state is given by $P(\epsilon)$. The other quantities in Eq. (2) are the Ricci tensor $R^{\kappa \sigma}$, the curvature scalar $R$, and the metric tensor, $g^{\kappa \sigma}$. No simple stability criteria are known for rapidly rotating stellar configurations in general relativity. However, an absolute limit on rapid rotation is set by the onset of mass shedding from the equator of a rotating star. The corresponding rotational frequency is known as the Kepler frequency, $\Omega_{\mathrm{K}}$. In classical mechanics, the expression for the Kepler frequency, determined by the equality between the centrifugal force 

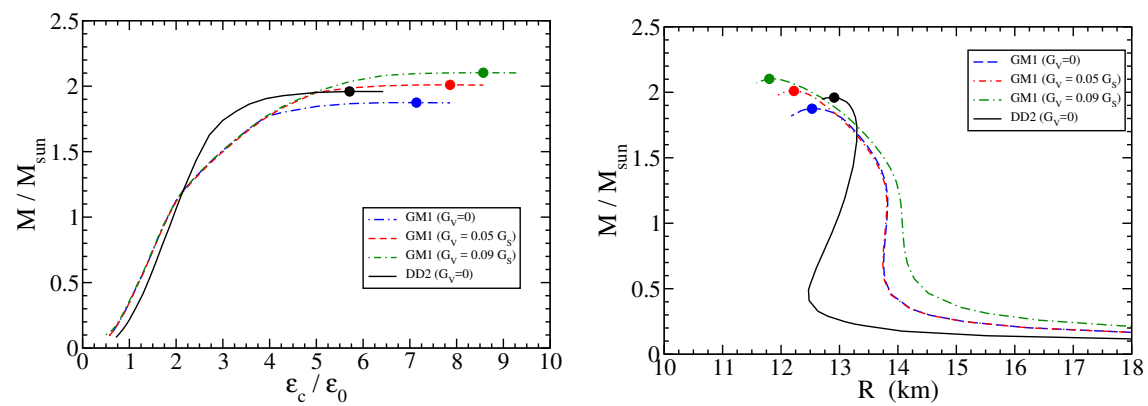

Fig. 1. Mass-central density (left) and mass-radius relationships of non-rotating neutron stars for several nuclear EoS. $\left(\epsilon_{0}=140 \mathrm{MeV} / \mathrm{fm}^{3}\right.$ denotes the density of infinite nuclear matter.) The solid dots mark the maximum-mass star of each stellar sequence. (Figure from Ref. 3.)

and gravity, is given by $\Omega_{\mathrm{K}}=\sqrt{M / R^{3}}$. Its general relativistic counterpart, which is obtained from $\delta \int d s^{2}=0$ evaluated in the star's equatorial plane, reads ${ }^{2,5}$

$$
\Omega_{\mathrm{K}}=\omega+\frac{\omega_{, r}}{2 \psi_{, r}}+e^{\nu-\psi} \sqrt{\frac{\nu_{, r}}{\psi_{, r}}+\left(\frac{\omega_{, r}}{2 \psi_{, r}} e^{\psi-\nu}\right)^{2}},
$$

where ${ }_{, r} \equiv \partial / \partial r$. Equation (3) is to be evaluated self-consistently at the equator of a rotating neutron star. The Kepler period follows from Eq. (3) as $P_{\mathrm{K}}=2 \pi / \Omega_{\mathrm{K}}$. For typical neutron star matter equations of state, the Kepler period obtained for $1.4 M_{\odot}$ neutron stars is typically around $1 \mathrm{~ms}^{1,2}$ An exception to this are strange quark matter stars. Since they are self-bound, they tend to possess smaller radii than neutron stars, which are bound by gravity only. Because of their smaller radii, strange stars can withstand mass shedding from the equator down to rotational periods of around $0.5 \mathrm{~ms}^{6,7}$

\section{Properties of Rotating Neutron Stars}

A mass increase of up to $\sim 20 \%$ is typical for rotation at $\Omega_{\mathrm{K}}$ (cf. Fig. 1). Because of rotation, the equatorial radii increase by several kilometers, while the polar radii become smaller by several kilometers. The ratio between both radii is around $2 / 3$, except for rotation close to the Kepler frequency. The most rapidly rotating, currently known neutron star is pulsar PSR J1748-2446ad, which rotates at a period of $1.39 \mathrm{~ms},(719 \mathrm{~Hz})^{8}$ well below the Kepler frequency for most neutron star equations of state ${ }^{1,2}$.

The density change in the core of a neutron star whose frequency varies from $0 \leq \Omega \leq \Omega_{\mathrm{K}}$ can be as large as $60 \% .^{2,10}$ This suggests that rotation may drive phase transitions and/or cause significant compositional changes of the matter in the cores of neutron stars ${ }^{1,2,10}$.

Figure 2 shows the mass versus central density relationships for both neutron stars spinning at their Kepler frequencies as well as non-rotating neutron stars. As one moves along either of these two lines, the baryon number changes, increasing 


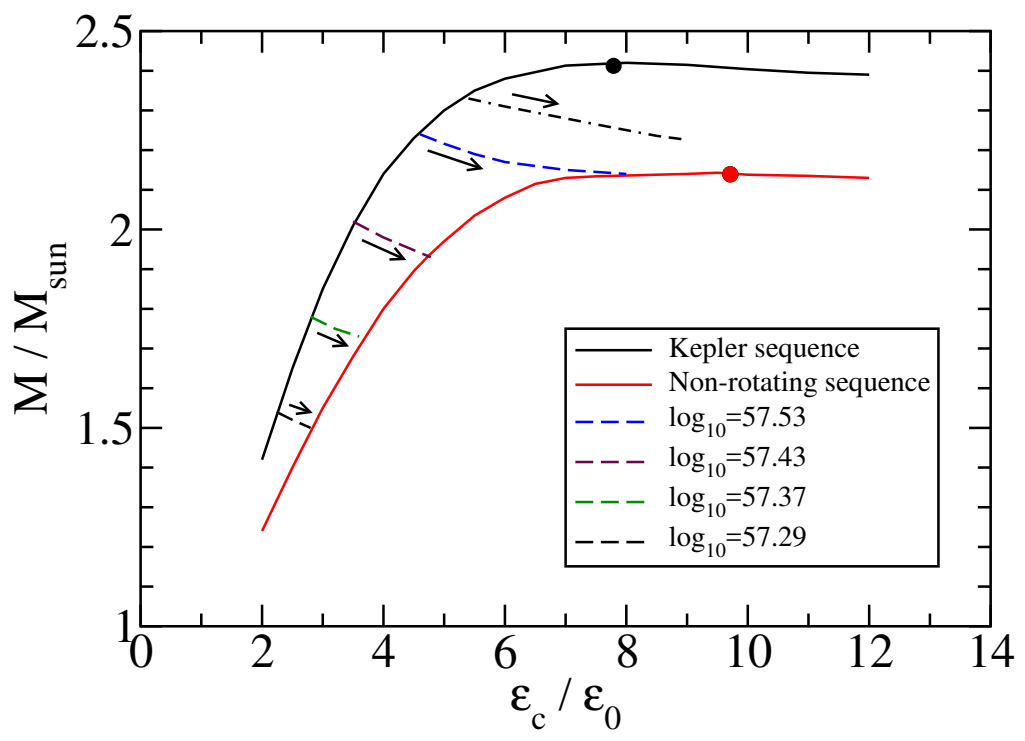

Fig. 2. Gravitational mass as a function of central stellar density of non-rotating and rotating neutron stars for the nuclear EoS GM1. Shown are several stellar paths (marked with arrows) that would be followed by neutron stars with a constant baryon number, $A$, as they spin down from their respective Kepler frequencies (black curve), $\Omega_{K}$, to zero frequency (red curve). (See text for more details.)

with larger central density. If one were to assume that a neutron star were secluded as it spins down (thus, not gaining or losing material), it would follow a path of constant baryon number, $A$, from the Kepler frequency curve down to the nonrotating one. Five such paths are depicted in Fig. 2. One sees that the Kepler frequency curve allows stable solutions with higher baryon numbers than there are stable solutions for the non-rotating case (dash-dotted curve in Fig. 2). Neutron stars of this type are called supra-massive rotating neutron stars (SURONS). ${ }^{3}$ It is thought that supra-massive rotating neutron stars collapse into black holes and have been a proposed source of fast radio bursts ${ }^{11}$.

Figures 3 and 4 show the quark-hadron compositions inside of rotating neutron stars. These models contain extended regions of matter consisting of a mixed phase of quarks and hadrons. This is a consequence of the fact that the Gibbs condition has been used to model the quark-hadron phase transition, in which case pressure varies monotonically with the proportion of the phases in equilibrium. This would not be the case if the Maxwell construction had been used to model the phase equilibrium between quarks and hadrons. Currently, it is an open issue which (if any of the two) descriptions characterizes the phase transition in the cores of neutron stars properly. Among other topics, this depends on the value of the surface tension of quark matter ${ }^{12}$. As can be seen, the quark-hadron mixed phase as well as several different hyperon species are successively spun out of the neutron star if the rotation rate increases toward the Kepler frequency. Non-rotating neutron stars posses the 
most complex compositions, since they are the most dense members of the stellar sequence. The compositions shown in Fig. 4 are snapshots taken from movies showing the entire rotational evolution of this neutron star from zero frequency all the way to $\Omega_{\mathrm{K}}$. The movies are publicly available ${ }^{13}$.

A heat map showing the quark-hadron content of rotating neutron stars computed for the GM1 equation of state is shown in Fig. 5. As can be seen from this figure, up to around $8 \%$ of the total gravitational mass of these neutron stars exists in the form a mixed quark-hadron phase. Lines of constant baryon number are also depicted in this figure as white lines, labeled with the logarithm of the stars' baryon number. These lines were included to give a sense of the path that a secluded neutron star would be expected to take as it spins down. By parsing out the maximum
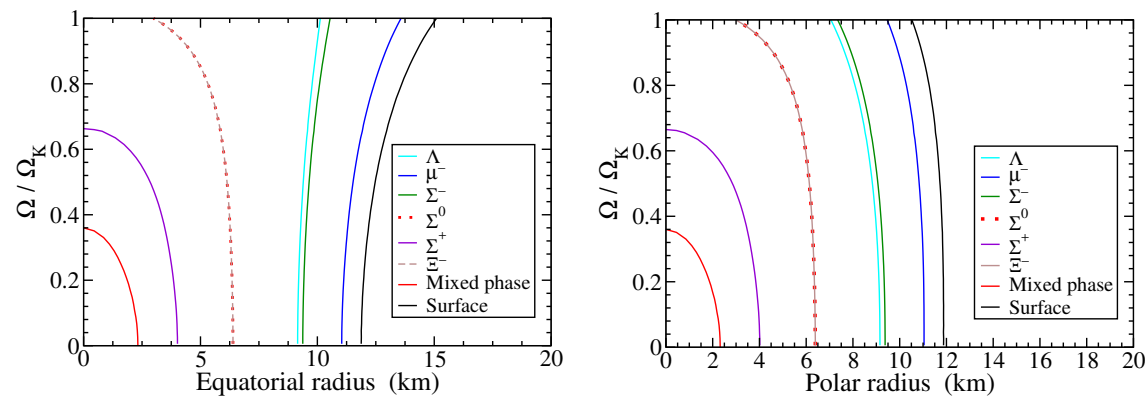

Fig. 3. Particle populations inside of rotating neutron stars, in equatorial (left) and polar (right) directions, computed for the GM1 EoS. The stellar frequency, $\Omega$, ranges from zero to the Kepler frequency, $\Omega_{\mathrm{K}}=1361 \mathrm{~Hz}$. The gravitational mass of the non-rotating star is $2.10 M_{\odot}$, which increases to $2.20 M_{\odot}$ for rotation at $\Omega=\Omega_{\mathrm{K}}$. (Figure from Ref. 3.)
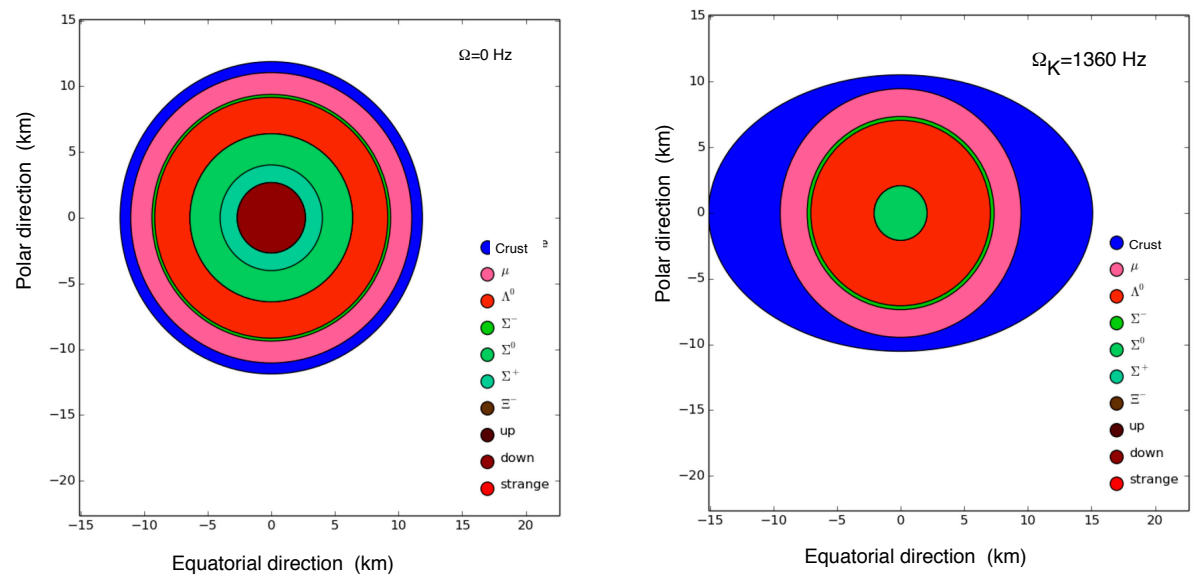

Fig. 4. Change of the composition of a $2 M_{\odot}$ neutron star caused by rotation, computed for the GM1 EoS. The star on the left (right) hand-side is non-rotating (rotating at the Kepler frequency, $\left.\Omega=\Omega_{\mathrm{K}}\right)$. The baryon number of both stars is the same $\left(\log _{10} A=57.51\right)$. (Figure from Ref. 3.) 


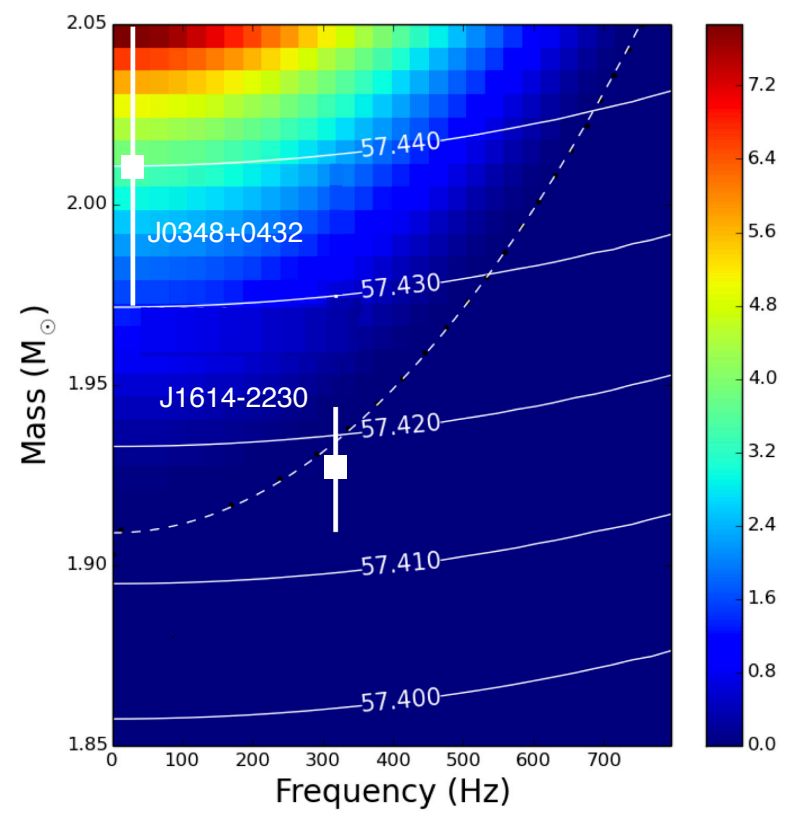

Fig. 5. Heat map showing the percent (column on the right) of total mass of a neutron star made up of deconfined quark matter, for the GM1 EoS. The white solid lines show the rotational evolution of neutron stars with constant baryon numbers $A$ (the reported figures being $\log _{10} A$ ). Also shown are the observed masses of pulsars J1614-2230 and J0348+0432 and the trend line (dashed white) fit, which separates confined from deconfined matter. (Figure from Ref. 3.)

frequency where deconfined quark matter is expected to exist at the center of the neutron star for a given mass, it is possible to get a curve through the gravitational mass-frequency diagram for the threshold above which one can expect to find deconfined quarks. These threshold frequencies for each mass were fit to determine a (quadratic) function for the curve. The fit equation, depicted as a dashed white line in Fig. 5, was found to have the form $M(\Omega)=a \Omega^{2}+c$, where $M$ is the neutron star's gravitational mass in solar masses, $\Omega$ its rotational frequency, and $a$ and $c$ are parameters. The values for $a$ and $c$ can be found in Ref. 3 .

Figure 5 allows one to estimate the amount of quark-hadron matter that may exist in the cores of neutron stars that have both a measured frequency and mass, as illustrated for pulsars PSR J1614-2230 $\left(M=1.928 \pm 0.017 M_{\odot}\right.$, rotational frequency $f=318 \mathrm{~Hz})^{14,15}$ and PSR J0348+0432 $\left(M=2.01 \pm 0.04 M_{\odot}, f=26 \mathrm{~Hz}\right)^{16,17}$ in Figure 5. According to this calculation, up to around $7 \%$ of the mass of PSR J0348+0432 could be in the mixed quark-hadron phase, while the core of the more rapidly rotating pulsars PSR J1614-2230 may be hovering right at the quark deconfinement density. 


\section{Summary}

The true nature of the matter deep in the cores of rotating neutron stars is still largely unknown despite several decades of intense research on this topic. In this short overview paper, we present the results of recent ${ }^{3}$ neutron star calculations performed for a non-local extension of the SU(3) Nambu-Jona-Lasinio (NJL) model to investigate the possible existence of deconfined quarks in the cores of neutron stars. As shown in this paper, the type and structure of the matter in the cores of rotating neutron stars depends sensitively on the star's spin frequency ${ }^{2,10,18}$. Exploring this feature in more details opens up a new window on the type of matter that exists in the central cores of neutron stars. We find that, depending on mass and rotational frequency, up to around $8 \%$ of the mass of massive neutron stars may be in the mixed quark-hadron phase, if the quark-hadron phase transition is modeled as a Gibbs phase transition. Examples of such massive neutron stars are pulsars PSR J1614-2230 with a gravitational mass of $1.928 \pm 0.017 M_{\odot}{ }^{14,15}$ and PSR J0348+0432 with a mass of $2.01 \pm 0.04 M_{\odot}{ }^{16,17}$ (Fig. 5). Pure quark matter in the centers of neutron stars is not obtained for any of the models for the nuclear equation that we have studied ${ }^{3}$. The gravitational mass at which quark deconfinement sets in in rotating neutron stars varies quadratically with spin frequency, which can be fitted by a simple quadratic formula. Owing to the unprecedented wealth of highquality data on pulsars provided by radio telescopes, X-ray satellites-and soon the latest generation of gravitational-wave detectors-it seems within reach to decode the inner workings of pulsars and, thus, decipher the phase diagram of cold and ultra-dense hadronic matter from astrophysics over the coming years.

\section{Acknowledgments}

This work is supported through the U.S. National Science Foundation under Grants PHY-1411708 and DUE-1259951. M. G. Orsaria acknowledges financial support from the American Physical Society's International Research Travel Award Program. G. A. Contrera and M. G. Orsaria acknowledge financial support from CONICET and UNLP (Project identification code 11/G140 and 11/X718), Argentina. Computing resources have been provided by the Computational Science Research Center and the Department of Physics at San Diego State University.

\section{References}

1. N. K. Glendenning, Compact Stars, Nuclear Physics, Particle Physics, and General Relativity, 2nd ed. (Springer-Verlag, New York, 2000).

2. F. Weber, Pulsars as Astrophysical Laboratories for Nuclear and Particle Physics, High Energy Physics, Cosmology and Gravitation Series (IOP Publishing, Bristol, Great Britain, 1999).

3. R. D. Mellinger, F. Weber, W. Spinella, G. A. Contrera, and M. G. Orsaria, Universe 3, 5 (2017).

4. G. A. Contrera, M. Orsaria, I. F. Ranea-Sandoval, and F. Weber, Hybrid Stars in the Framework of different NJL Models., arXiv:1612.09485 [nucl-th](2016). 
5. J. L. Friedman, J. R. Ipser, and L. Parker, Astrophys. J. 304, 115 (1986).

6. N. K. Glendenning and F. Weber, Astrophys. J. 400, 647 (1992).

7. N. K. Glendenning, Phys. Rev. D 46, 4161 (1992).

8. J. W. T. Hessels et al., Science 1901, 311 (2006).

9. J. W. T. Hessels, S. M. Ransom, I. H. Stairs, P. C. C. Freire, V. M. Kaspi, and F. Camilo, Science 311, 1901 (2006).

10. F. Weber, Prog. Part. Nucl. Phys. 54, 193 (2005).

11. H. Falcke and L. Rezzolla, A\&A 562, A137 (2014).

12. N. Yasutake et al., Phys. Rev. C 89065803 (2014); L. F. Palhares and E. S. Fraga, Phys. Rev. D 82, 125018 (2010); M. B. Pinto, V. Koch, and J. Randrup, Phys. Rev. C 86, 025203 (2012); B. W. Mintz, R. Stiele, R. O. Ramos, and J. Schaffner-Bielich, Phys. Rev. D 87, 036004 (2013).

13. http://www-rohan.sdsu.edu/fweber/Mellinger/Compositions.html.

14. P. B. Demorest, T. Pennucci, S. M. Ranson, M. S. E. Roberts, and J. W. T. Hessels, Nature 467, 1081 (2010).

15. E. Fonseca et al., Astrophys. J. 832, 2 (2016).

16. R. S. Lynch et al., Astrophys. J. 81, 763 (2013),

17. J. Antoniadis et al., Science 340, 6131 (2013).

18. M. Stejner, F. Weber, and J. Madsen, Astrophys. J. 694, 1019 (2009). 